\title{
Analgesia Pós-Operatória com Bloqueio Bilateral do Nervo Pudendo com Bupivacaína S75:R25 a 0,25\%. Estudo Piloto em Hemorroidectomia sob Regime Ambulatorial *
}

\section{Bilateral Pudendal Nerves Block for Postoperative Analgesia with $0.25 \%$ S75:R25 Bupivacaine. Pilot Study on Outpatient Hemorrhoidectomy}

\author{
Luiz Eduardo Imbelloni, TSA ${ }^{1}$; Lúcia Beato, TSA ${ }^{2}$; Carolina Beato ${ }^{2}$; José Antônio Cordeiro ${ }^{3}$; Dulcimar Donizete de Souza ${ }^{4}$
}

\begin{abstract}
RESUMO
Imbelloni LE, Beato L, Beato C, Cordeiro JA, Souza DD - Analgesia Pós-Operatória com Bloqueio Bilateral do Nervo Pudendo com Bupivacaína S75:R25 a 0,25\%. Estudo Piloto em Hemorroidectomia sob Regime Ambulatorial
\end{abstract}

JUSTIFICATIVA E OBJETIVOS: A hemorroidectomia pode ser realizada sob várias técnicas anestésicas e em regime torno para a residência. O objetivo deste estudo foi avaliar as vantagens e a realização do bloqueio bilateral dos nervos pudendos para analgesia pós-operatória em hemorroidectomias.

MÉTODO: O bloqueio bilateral dos nervos pudendos com bupivacaína S75:R25 a 0,25\% foi realizado com estimulador de nervos em 35 pacientes submetidos à hemorroidectomia sob raquianestesia. Foram avaliadas intensidade da dor, duração da analgesia, analgesia de demanda e eventuais complicações relacionadas à técnica. Os dados foram avaliados às $6,12,18$, 24 e 30 horas após o término da intervenção cirúrgica.

RESULTADOS: Em todos os pacientes, foi obtido sucesso com a estimulação de ambos os nervos pudendos. Em nenhum momento da avaliação ocorreu dor intensa. Até 12 horas após o bloqueio, todos os pacientes apresentaram anestesia na região perineal; com 18 horas, 17 pacientes e 24 horas; 10 pacientes $A$ analgesia pós-operatória foi ótima em 18 pacientes; satisfatória, em cinco pacientes; e insatisfatória, em sete pacientes. A duração média da analgesia foi de 23,77 horas. Não ocorreram alterações da pressão arterial, da freqüência cardiaca, nem foram observadas náuseas ou vômitos. Todos os pacientes tiveram micção espontânea. Nenhuma complicação local ou sistêmica foi relacionada ao anestésico local. Vinte e sete pacientes classificaram de excelente a técnica de analgesia e apenas três pacientes do sexo masculino ficaram satisfeitos o que foi justificado pela anestesia no pênis.

\footnotetext{
${ }^{*}$ Recebido do (Received from) Instituto de Anestesia Regional, São José do Rio Preto, SP

1. Diretor do Instituto de Anestesia Regional, Hospital de Base, São José do Rio Preto, Anestesiologista da Clínica São Bernardo, Rio de Janeiro, RJ

2. Anestesiologista da Clínica São Bernardo, Rio de Janeiro, RJ

3. Professor Livre Docente em Probabilidade e Estatística da Faculdade de Medicina de São José do Rio Preto, SP

4. Doutor em Ciências da Saúde; Diretor Adjunto de Pessoal da Faculdade de Medicina de São José do Rio Preto, SP

Apresentado (Submitted) em 28 de abril de 2005

Aceito (Accepted) para publicação em 06 de julho de 2005

Endereço para correspondência (Correspondence to)

Dr. Luiz Eduardo Imbelloni

Av. Epitácio Pessoa, 2356/203 Lagoa

22471-000 Rio de Janeiro, RJ

E-mail:dr.imbelloni@terra.com.br
}

(C) Sociedade Brasileira de Anestesiologia, 2005 ambulatorial. A dor pós-operatória é intensa e pode atrasar o re-

CONCLUSÕES: O bloqueio bilateral dos nervos pudendos, orientado por estimulador de nervos, proporciona a analgesia de excelente qualidade, com baixa necessidade de opióides, sem complicações local ou sistêmica e sem retenção urinária. Estudos controlados permitirão demonstrar se esta técnica deve ser a primeira opção para analgesia em hemorroidectomias. A permanência de anestesia perineal por 20,21 horas deverá induzir novos trabalhos com o bloqueio dos nervos pudendos orientado por estimulador para o ato cirúrgico.

Unitermos: ANESTÉSICOS, Local: bupivacaína, mistura com excesso enantiomérico (S75:R25); CIRURGIA, Anorretal: hemorroidectomia; TÉCNICAS ANESTÉSICAS, Regional: bloqueio pudendo

\section{SUMMARY}

Imbelloni LE, Beato L, Beato C, Cordeiro JA, Souza DD - Bilateral Pudendal Nerves Block for Postoperative Analgesia with $0.25 \%$ S75:R25 Bupivacaine. Pilot Study on Outpatient Hemorrhoidectomy

BACKGROUND AND OBJECTIVES: Hemorrhoidectomy may be performed under several anesthetic techniques and in outpatient regimen. Postoperative pain is severe and may delay discharge. This study aimed at evaluating bilateral pundendal nerves block for post- hemorrhoidectomy analgesia.

METHODS: Bilateral pundendal nerves block with $0.25 \%$ S75:R25 bupivacaine was performed with nerve stimulator in 35 patients submitted to hemorrhoidectomy under spinal anesthesia. Evaluated parameters were pain severity, duration of analgesia, demand analgesia and possible technique-related complications. Data were evaluated 6, 12, 18, 24 and 30 hours after surgery completion.

RESULTS: Successful pudendal nerves stimulation was achieved in all patients. There has been no severe pain in all evaluated moments. At 12 hours after blockade, all patients had perineal anesthesia; at 18 hours, 17 patients and at 24 hours, 10 patients still presented perineal anesthesia. Postoperative analgesia was optimal for 18 patients; satisfactory, for 5 patients; and unsatisfactory, for 7 patients. Mean analgesic duration was 23.77 hours. There were no changes in blood pressure, heart rate, no nausea and vomiting were observed. All patients had spontaneous micturition. No local anesthetic-related local or systemic complications were observed. Technique was considered excellent by 27 patients and only 3 male patients considered it satisfactory due to penile anesthesia.

CONCLUSIONS: Bilateral pudendal nerves block oriented by nerve stimulator provides excellent analgesia with low need for opioids, without local or systemic complications and without urinary retention. Controlled studies might be able to show whether this should be the first analgesic option for hemorrhoidectomies. Perineal anesthesia lasting 20.21 hours shall induce further studies with stimulator-oriented pudendal block.

Key Words: ANESTHETICS, Local: bupivacaine, enantiomeric mixture (S75:R25); ANESTHETIC TECHNIQUES, Regional: pudendal block; SURGERY, Anorectal: hemorrhoidectomy 


\section{INTRODUÇÃO}

doença hemorroidária é comum no mundo inteiro e causa sintomas em $4,4 \%$ da população ${ }^{1}$. Noventa por cento das cirurgias anorretais podem ser realizadas em regime ambulatorial $^{2}$. Entre todas as modalidades de tratamento da doença hemorroidária, a ressecção cirúrgica parece ser a que melhor elimina os sintomas e proporciona uma melhor qualidade de vida desses pacientes ${ }^{3}$. Entretanto, a intensa dor pós-operatória pode aumentar a necessidade de internação hospitalar ${ }^{4}$.

A dor pós-operatória é um dos maiores problemas em pacientes ambulatoriais ${ }^{5}$. Estudo retrospectivo com 1100 pacientes submetidos à cirurgia sob regime ambulatorial mostrou que $35 \%$ dos pacientes sentiam dor - de moderada à intensa - em suas residências, apesar do uso de terapia analgésica ${ }^{6}$. Diversos métodos analgésicos têm sido preconizados para alívio da dor pós-operatória em cirurgias de hemorróidas como a morfina subcutânea com bomba de infusão ${ }^{7}$, a estimulação elétrica transcutânea ${ }^{8}$, a dexametasona ${ }^{9}$, a infiltração perianal com bupivacaína ${ }^{10}$, o bloqueio posterior do períneo ${ }^{11}$ e o bloqueio da fossa isquiorretal ${ }^{12}$.

O nervo pudendo é formado pelos ramos posteriores de $S_{2}$, $\mathrm{S}_{3}$ e $\mathrm{S}_{4}$ e se divide em quatro ramos, nervos anais inferiores, nervos perineais, nervos labiais posteriores (mulher), nervos escrotais posteriores (homem), nervo dorsal do clitóris (mulher) e nervo dorsal do pênis (homem) ${ }^{13}$. Em teoria o bloqueio do nervo pudendo pode proporcionar analgesia ou anestesia da região perineal, sendo freqüentemente realizado por cirurgiões ou obstetras. O estimulador de nervos periféricos, que é um excelente método de ensino da anestesia regional, facilitou ao anestesiologista, a realização desse tipo de bloqueio, através de sua localização monitorizada pela contração da musculatura perineal.

Diversos estudos em animais e humanos têm demonstrado que os anestésicos locais levógiros são menos tóxicos para o sistema nervoso central e cardiovascular do que a bupivacaína racêmica ou dextrógira, e que exibem uma capacidade intrínseca de produzir vasoconstrição e menor bloqueio motor 14,15. Comparando a bupivacaína racêmica com a bupivacaína em excesso enantiomérico (S75:R25) em bloqueio do plexo braquial, não foi observada diferença nos parâmetros observados ${ }^{16}$. Os autores concluem sugerindo que a S75:R25 representa uma alternativa mais segura em relação à bupivacaína racêmica devido sua menor toxicidade ${ }^{16}$.

O objetivo deste estudo foi avaliar a analgesia pós-operatória em hemorroidectomia com a bupivacaína S75:R25 a $0,25 \%$ injetada bilateralmente nos nervos pudendos localizados com a técnica de estimulação elétrica.

\section{MÉTODO}

Após aprovação do Comitê de Ética e consentimento informado, participaram deste estudo 35 pacientes, estado físico ASA I e II, com idade entre 20 e 60 anos, submetidos à raquianestesia com 50 mg de lidocaína a $2 \%$ isobárica para hemorroidectomia em regime ambulatorial. A raquianestesia foi re- alizada em decúbito lateral esquerdo no espaço $L_{3}-L_{4}$, usando a via paramediana com agulha 27G Quincke (B. Braun Melsungen S.A.). A monitorização consistiu de medidas da pressão arterial por método não-invasivo, freqüência cardíaca e oxímetro de pulso. Não foi administrada medicação pré-anestésica. Após a chegada à sala de operação, foi instalada venóclise com solução de Ringer com lactato e administrados 50 a $100 \mu \mathrm{g}$ de fentanil, por via venosa. Quantidades mínimas de líquidos foram infundidas no intra-operatório, sempre menores que $500 \mathrm{~mL}$.

No final da cirurgia o bloqueio bilateral dos nervos pudendos foi realizado com o paciente em posição de litotomia, ainda sob o efeito da raquianestesia. $\mathrm{O}$ acesso foi transperineal e o local de punção foi medial à tuberosidade isquiática em cada lado, utilizando agulha isolada de $100 \mathrm{~mm}$ (B. Braun Melsungen AG, agulha $21 \mathrm{G} 0,8 \times 100 \mathrm{~mm}$ ) conectada a um estimulador de nervo periférico (Stimuplex ${ }^{\circledR}, B$. Braun Melsungen AG) regulado para liberar uma corrente pulsátil quadrada de 1 $\mathrm{mA}$, com freqüência de $2 \mathrm{~Hz}$, inserida perpendicularmente, numa profundidade de aproximadamente $7 \mathrm{~cm}$, procurando obter contração do esfíncter anal. Obtida contração perineal, $20 \mathrm{~mL}$ de mistura com excesso enantiomérico (S75:S25) de bupivacaína a $0,25 \%$ foram injetados em cada lado.

Os pacientes foram acompanhados nas primeiras seis horas no hospital e posteriormente às 12, 18, 24 e 30 horas por telefone, quando eram questionados em relação à intensidade de dor, que era classificada em: sem dor (grau 0), leve (grau 1), moderada (grau 2) ou intensa (grau 3). Perguntava-se, também, se a região operada estava dormente (anestesia). Avaliada a dor da primeira evacuação, usando-se a mesma escala de dor, anotou-se o tempo decorrido entre o bloqueio e a evacuação. Os pacientes opinaram sobre a analgesia pós-operatória escolhendo um dos termos apresentados: excelente, satisfatória ou ruim.

O tramadol (50 mg) por via oral foi prescrito em caso de dor. A analgesia foi classificada em ótima se não houvesse necessidade de analgésico (tramadol); satisfatória (uma dose) e insatisfatória (duas ou mais doses). Dados demográficos, período livre de dor, intensidade de dor, freqüência de analgésico por via oral, total de doses administradas e complicações como retenção urinária foram avaliados.

As variáveis qualitativas (existência de dor, intensidade da dor, dor na primeira evacuação, bloqueio em 6, 12, 18, 24 e 30 horas, e grau de satisfação) foram analisadas, em relação ao sexo, pelo teste Exato de Fisher. Variáveis quantitativas comparadas em relação ao tempo foram analisadas pelo teste t pareado, e em relação aos sexos pelo teste t para duas amostras presumindo variâncias diferentes.

\section{RESULTADOS}

Os dados demográficos dos pacientes estão apresentados na tabela I. Em todos os pacientes a raquianestesia foi satisfatória para o procedimento e nenhum paciente necessitou de complementação com anestesia geral. Em todos os pacientes foi obtido sucesso na estimulação de ambos os nervos pudendos. Não ocorreram alterações da pressão arterial, da 
freqüência cardíaca nem foram observadas náuseas ou vômitos no pós-operatório. Todos os pacientes tiveram micção espontânea. Nenhuma complicação local ou sistêmica foi relacionada ao anestésico local.

Tabela I - Dados Demográficos

\begin{tabular}{lc}
\hline Variáveis & \\
\hline Idade $(\mathrm{anos})^{*}$ & $44,65 \pm 8,09$ \\
Peso $(\mathrm{kg})^{*}$ & $69,68 \pm 11,20$ \\
Altura $(\mathrm{cm})^{*}$ & $167,71 \pm 8,57$ \\
Sexo & \\
$\quad$ Feminino & 18 \\
$\quad$ Masculino & 17 \\
\hline
\end{tabular}

* Valores expressos em Média \pm DP

O resultado da pesquisa da intensidade da dor durante as primeiras 30 horas do pós-operatório está na tabela ll e em momento algum do estudo ocorreu dor máxima (intensa). Até 12 horas após o bloqueio todos os pacientes apresentam anestesia na região perineal; com 18 horas, 22 pacientes; com 24 horas, 12 pacientes e com 30 horas, nenhum paciente (Tabela II).

Tabela II - Escala de Dor nos Diferentes Momentos e Pacientes com Anestesia na Região Perineal (ânus e vulva)

\begin{tabular}{lccccc}
\hline Momentos $(\mathrm{h})$ & \multicolumn{4}{c}{ Escala de Dor } & $\begin{array}{c}\text { Anestesia } \\
\text { Períneo }\end{array}$ \\
\cline { 2 - 5 } & Sem & Leve & Moderada & Intensa & \\
\hline 6 & 31 & 4 & 0 & 0 & 35 \\
12 & 31 & 4 & 0 & 0 & 35 \\
18 & 24 & 11 & 0 & 0 & 22 \\
24 & 23 & 9 & 3 & 0 & 12 \\
30 & 04 & 26 & 0 & 0 & 0 \\
\hline
\end{tabular}

As 6 e 12 horas de pós-operatório nenhuma mulher relatou dor leve e quatro homens a relataram (os restantes não relataram dor), mostrando haver evidência de maior incidência de dor nesses momentos nos homens. Às 18 horas, uma muIher relatou dor leve, o que ocorreu em 10 homens, havendo, portanto, evidência de maior dor dentre os homens. Às 24 horas, duas mulheres relataram dor moderada e nenhuma dor leve, enquanto entre os homens ocorreu um caso de dor moderada e nove de dor leve. Isso evidencia maior probabilidade de dor leve ou moderada entre os homens. Duas mulheres relataram dor na primeira evacuação no pós-operatório, enquanto entre os homens, ocorreu em oito pacientes. Até 12 horas após o bloqueio, todos os pacientes apresentaram anestesia na região perineal; com 18 horas, 22 pacientes, 15 mulheres e 7 homens, indicando haver evidência de menor probabilidade de bloqueio dentre os homens nesse período. Com 24 horas o bloqueio persistia em 12 pacientes, 5 mulheres e 7 homens, não havendo diferença estatística significativa nesse momento.
A duração da analgesia foi de 15 a 20 horas em 10 pacientes; de 21 a 25 horas, em 14 pacientes; de 26 a 30 horas; em 10 pacientes; mais de 31 horas em um paciente, com média de 23,77 horas. A analgesia pós-operatória foi ótima em 23 pacientes; satisfatória, em cinco pacientes, e insatisfatória, em sete pacientes. Em 23 pacientes não foi necessário o uso de analgésico no pós-operatório. A primeira evacuação ocorreu em torno de 30 horas após o bloqueio bilateral dos pudendos, sendo que 10 pacientes relataram dor durante a evacuação, enquanto que 25 pacientes não relataram dor. Trinta e dois pacientes classificaram de excelente a técnica de analgesia e apenas três pacientes do sexo masculino ficaram satisfeitos, o que é justificado pela anestesia no pênis. Não há evidência de diferença no grau de satisfação segundo o sexo $(p=0,10)$ (Tabelas III e IV).

Tabela III - Duração da Analgesia, Duração da Anestesia no Períneo, Tempo para Primeira Evacuação, Dor à Primeira Evacuação e Satisfação com a Técnica

\begin{tabular}{|c|c|}
\hline Duração da analgesia $(h)^{*}$ & $23,77 \pm 4,49$ \\
\hline Duração da anestesia períneo $(h)^{*}$ & $20,21 \pm 2,79$ \\
\hline Tempo da $1^{\mathrm{a}}$ evacuação $(\mathrm{h})^{*}$ & $29,60 \pm 4,84$ \\
\hline \multicolumn{2}{|l|}{ Primeira evacuação } \\
\hline Com dor & 10 \\
\hline Sem dor & 25 \\
\hline \multicolumn{2}{|l|}{ Satisfação com a técnica } \\
\hline Excelente & 32 \\
\hline Satisfatória & 3 \\
\hline Ruim & 0 \\
\hline \multicolumn{2}{|l|}{ * Valores expressos pela Média \pm DP } \\
\hline \multicolumn{2}{|c|}{ Tabela IV - Duração da Analgesia e Número de Paciente } \\
\hline 16 & 1 \\
\hline 17 & 1 \\
\hline 18 & 1 \\
\hline 19 & 6 \\
\hline 20 & 1 \\
\hline 21 & 3 \\
\hline 22 & 1 \\
\hline 23 & 1 \\
\hline 24 & 3 \\
\hline 25 & 6 \\
\hline 26 & 3 \\
\hline 28 & 3 \\
\hline 29 & 1 \\
\hline 30 & 3 \\
\hline 36 & 1 \\
\hline $23,77 \pm 4,49$ & \\
\hline
\end{tabular}




\section{DISCUSSÃO}

A trombose hemorroidária externa é provavelmente um dos mais freqüentes diagnósticos de emergência anorretal e o tratamento cirúrgico, o de eleição. Neste estudo a analgesia pós-operatória com bloqueio bilateral dos pudendos sob raquianestesia resultou em analgesia de duração média de 23,77 horas e baixa necessidade de complementação com opióides, com 23 pacientes sem medicação de resgate.

A hemorroidectomia é uma cirurgia de curta duração, entretanto extremamente dolorosa e poucos estudos são direcionados à analgesia pós-operatória ${ }^{17}$. Os primeiros fatores da dor estão ligados ao ato cirúrgico. A técnica de Milligan-Morgan (aberta) parece ser muito mais dolorosa que a hemorroidectomia semi-aberta de Reis Neto ou mesmo que a fechada

18. Certos autores utilizam o bloqueio perineal posterior como técnica de anestesia e analgesia pós-operatória. Existem diversas variantes das descrições iniciais ${ }^{11,19,20}$. As diferenças dizem respeito aos detalhes técnicos da realização, dos equipamentos e anestésicos utilizados. De outra forma, é necessário fazer distinção entre infiltração pararretal e bloqueio perineal. A infiltração pararretal (ou perineal) corresponde somente ao modo superficial do bloqueio perineal posterior e este é freqüentemente realizado como técnica única ${ }^{21}$. No presente trabalho, foi utilizado o bloqueio bilateral dos nervos pudendos orientado por estimulador de nervos, que resultou em anestesia da região perineal por 20,21 horas, mostrando que o bloqueio pode ser usado como técnicaúnica para a cirurgia em questão. Não foi proposta do estudo avaliar a diferença na incidência de dor em ambos os sexos. Entretanto, encontrou-se maior incidência de dor nos homens do que nas mulheres em todos os períodos de avaliação e durante a primeira evacuação. Este dado deverá ser objeto de posteriores estudos.

Todos os anestésicos já foram utilizados em bloqueio perineal posterior: bupivacaína, lidocaína, mepivacaína, ropivacaína, associados ou não entre si, com ou sem epinefrina e com volumes variando de 20 a $50 \mathrm{~mL}$. Utilizando lidocaína ou mepivacaína em 400 pacientes, a duração da analgesia foi de 5 horas em $31,5 \%$; mais de 10 horas em $48,5 \%$ e mais de 15 horas em $9,2 \%$, sendo que $3,2 \%$ não fizeram uso de qualquer analgésico ${ }^{11}$. Um a três $\mathrm{mL}$ de bupivacaína a $0,5 \%$ injetado na base de cada hemorróida, 10 minutos antes da incisão, proporcionou um período sem dor 10 vezes maior do que o grupo controle ${ }^{10}$. Ainjeção de $20 \mathrm{~mL}$ de mistura com excesso enantiomérico (S75:R25) de bupivacaína a 0,25\% em cada nervo pudendo proporcionou uma analgesia média de 23,77 horas de duração. Em outra publicação ${ }^{16}$, que avaliou a mesma solução com o dobro da concentração $(0,5 \%)$ no plexo braquial, não foi observado o tempo de analgesia, impossibilitando a comparação dos resultados.

A raquianestesia causa distúrbios clínicos da função vesical devido à interrupção do reflexo da micção. A função da bexiga permanece imperfeita até que o bloqueio tenha regredido ao $3^{\circ}$ segmento sacral em todos os pacientes ${ }^{22}$. Com anestésicos de longa duração, o volume acumulado pode exceder a capacidade cistométrica da bexiga ${ }^{17}$. Por esta razão, a ra- quianestesia neste estudo foi realizada com 50 mg de lidocaína a $2 \%$ isobárica, na tentativa de evitar retenção urinária devido à técnica anestésica para a cirurgia, fato confirmado com a micção espontânea em todos os pacientes. Este dado demonstra que o bloqueio do nervo pudendo bilateral cursou com anestesia na região perineal por praticamente 20 horas e não impediu a micção espontânea.

Este estudo piloto utilizou a estimulação bilateral dos nervos pudendos com emprego de um estimulador de nervos periféricos para controle da dor pós-operatória de pacientes submetidos à hemorroidectomia sob raquianestesia. O estudo tem suas limitações em não comparar o resultado da analgesia com outro método.

Os autores, com base nos resultados desse estudo, consideram que o bloqueio bilateral dos nervos pudendos com estimulador de nervos periféricos para analgesia pós-operatória com bupivacaína S75:R25 a 0,25\% pode ser indicado em todos casos de cirurgia anorretal, quando não houver infecção prévia. Com esta técnica a analgesia é eficiente, sem complicações local ou sistêmica, tornando a primeira evacuação menos dolorosa e sem efeitos colaterais, como a retenção urinária. No futuro, estudos controlados, e comparativos, permitirão demonstrar se esta técnica deve ser a primeira opção para analgesia em hemorroidectomias. A permanência de anestesia perineal por 20,21 em média horas deverá induzir novos trabalhos com o bloqueio dos pudendos com estimulador para o ato cirúrgico.

\section{AGRADECIMENTO}

Ao professor Manoel Antônio Pereira Alvarez pela sugestão do trabalho.

\section{Bilateral Pudendal Nerves Block for Postoperative Analgesia with $0.25 \%$ S75:R25 Bupivacaine. Pilot Study on Outpatient Hemorrhoidectomy}

Luiz Eduardo Imbelloni, TSA, M.D.; Lúcia Beato, TSA, M.D.; Carolina Beato, M.D.; José Antônio Cordeiro, M.D.; Dulcimar Donizete Souza, M.D.

\section{INTRODUCTION}

Hemorrhoidal diseases are common throughout the world and are symptomatic in $4.4 \%$ of the population ${ }^{1}$. Approximately $90 \%$ of anorectal procedures may be performed in outpatient regimen ${ }^{2}$. Among all treatments for hemorrhoidal diseases, surgical resection seems to be the best to eliminate symptoms and improve quality of life ${ }^{3}$. However, severe post- 
operative pain may prolong hospital stay ${ }^{4}$. Postoperative pain is a major outpatient regimen problem ${ }^{5}$. A retrospective study with 1100 patients submitted to outpatient procedures has shown that $35 \%$ of patients had moderate to severe pain at home, in spite of analgesic therapy ${ }^{6}$. Several analgesic methods have been proposed for post-hemorrhoidectomy pain relief, such as subcutaneous morphine with infusion pump ${ }^{7}$, transcutaneous electric stimulation ${ }^{8}$, dexametazone ${ }^{9}$, perianal infiltration with bupivacaine ${ }^{10}$, posterior perineal block ${ }^{11}$ and of the ischiorectal fossa ${ }^{12}$. The pudendal nerve is formed by posterior $S_{2}, S_{3}$ and $S_{4}$ branches and is divided in four branches: inferior anal nerves, perineal nerves, posterior labial nerves (females), posterior scrotal nerves (males), clitoris dorsal nerve (females) and penile dorsal nerve (male $)^{13}$. In theory, pudendal nerve block may provide perineal analgesia or anesthesia being often used by surgeons and obstetricians. Peripheral nerve stimulator, which is an excellent teaching method for regional anesthesia, helps the anesthesiologist in this type of blockade due to location monitored by perineal muscles contracture.

Different animal and human studies have shown that levogyrous local anesthetics are less toxic for central nervous and cardiovascular systems as compared to racemic or dextrogyrous bupivacaine, with intrinsic ability to promote vasoconstriction and less intense motor block ${ }^{14,15}$. A comparison of racemic and bupivacaine with levogyrous enantiomeric excess of $50 \%(S 75 ; R 25)$ for brachial plexus block, has not shown differences in observed parameters ${ }^{16}$. Authors concluded suggesting that $S 75: R 25$ is a safer alternative as compared to racemic bupivacaine due to lower toxicity ${ }^{16}$.

This study aimed at evaluating post-hemorrhoidectomy analgesia with $0.25 \%$ S75:R25 bupivacaine bilaterally injected in pudendal nerves located with the aid of electrical stimulation.

\section{METHODS}

After the Ethics Committee approval and their informed consent, participated in this study 35 patients physical status ASA I and II, aged 20 to 60 years, submitted to spinal anesthesia with $50 \mathrm{mg}$ of $2 \%$ isobaric lidocaine for hemorrhoidectomy in outpatient regimen. Spinal anesthesia was induced in the left lateral position in $L_{3}-L_{4}$ interspace, by the paramedial route with 27G Quincke needle (B. Braun Melsungen S.A.). Monitoring consisted of noninvasive blood pressure, heart rate and pulse oximetry. Patients were not premedicated. Venoclysis was installed in the operating room with lactated Ringer's and 50 to $100 \mu \mathrm{g}$ fentanyl were intravenously administered. Minimum intraoperative fluids were administered, always below $500 \mathrm{~mL}$.

Bilateral pudendal nerves block was performed at surgery completion with patients in lithotomy position and under spinal anesthesia effect. The needle access was transperineal and medial to ischial tuberosity on both sides, using beveled insulated needle with 100 mm (B. Braun Melsungen AG, 21G
$0.8 \times 100 \mathrm{~mm}$ needle) connected to peripheral nerve stimulator (Stimuplex ${ }^{\circledR}, B$. Braun Melsungen AG) set to release a square pulsatile current of $1 \mathrm{~mA}$, with $2 \mathrm{~Hz}$ frequency, perpendicularly inserted to a depth of approximately $7 \mathrm{~cm}$, trying to obtain anal sphincter contraction. After perineal contraction, $20 \mathrm{~mL}$ of $0.25 \%$ bupivacaine (S75:R25) were injected in each side.

Patients were followed for six hours in the hospital and then at $12,18,24$ and 30 hours by telephone when they were questioned about pain severity, which was classified as: absent (level 0), mild (level 1), moderate (level 2) or severe (level 3). Patients were also asked whether the operated region was insensitive (anesthesia). Pain at first evacuation was evaluated with the same scale and time between blockade and first evacuation was recorded. Patients were asked about postoperative analgesia, which should be classified as excellent, satisfactory or poor.

Oral tramadol was prescribed in case of pain. Analgesia was classified as optimal if no analgesic (tramadol) was needed; satisfactory (one dose) and unsatisfactory (two or more doses). Demographics data, painless period, pain severity, oral analgesic frequency, total doses and complications, such as urinary retention, were evaluated.

Qualitative variables (presence of pain, pain severity, pain at first evacuation, blockade at 6, 12, 18, 24 and 30 hours and level of satisfaction) were evaluated with regard to gender by Fisher's Exact test. Quantitative variables compared with regard to time were analyzed by paired t test, and with regard to gender by t test for two samples, assuming different variances.

\section{RESULTS}

Demographics data are shown in table I. Spinal anesthesia was satisfactory for all patients and no patient needed complementation with general anesthesia. Both pudendal nerves were successfully stimulated in all patients. There were no changes in blood pressure and heart rate, there were no postoperative nausea or vomiting. All patients had spontaneous micturition. No local or systemic complications were related to local anesthetics.

Table I - Demographics Data

\begin{tabular}{|c|c|}
\hline Variables & \\
\hline Age (years)* & $44.65 \pm 8.09$ \\
\hline Weight $(\mathrm{kg})^{*}$ & $69.68 \pm 11.20$ \\
\hline Height $(\mathrm{cm})$ * & $167.71 \pm 8.57$ \\
\hline \multicolumn{2}{|l|}{ Gender } \\
\hline Female & 18 \\
\hline Male & 17 \\
\hline
\end{tabular}

${ }^{*}$ Values expressed in Mean \pm SD 
Pain severity in the first 30 postoperative hours is shown in table Il and there has been no maximum pain (severe) throughout the study. All patients presented perineal anesthesia 12 hours after blockade. At 18 hours, 22 patients; at 24 hours, 12 patients and at 30 hours, no patient presented perineal anesthesia (Table II).

Table II - Pain Scale in Different Moments and Patients with Perineal Anesthesia (anus and vulva)

\begin{tabular}{|c|c|c|c|c|c|}
\hline \multirow[t]{2}{*}{ Moments (h) } & \multicolumn{4}{|c|}{ Pain Scale } & \multirow{2}{*}{$\begin{array}{l}\text { Anesthesia } \\
\text { Perineum }\end{array}$} \\
\hline & Without & Mild & Moderate & Severe & \\
\hline 6 & 31 & 4 & 0 & 0 & 35 \\
\hline 12 & 31 & 4 & 0 & 0 & 35 \\
\hline 18 & 24 & 11 & 0 & 0 & 22 \\
\hline 24 & 23 & 9 & 3 & 0 & 12 \\
\hline 30 & 04 & 26 & 0 & 0 & 0 \\
\hline
\end{tabular}

At 6 and 12 postoperative hours no female has referred mild pain and four males have referred it (remaining males did nor refer pain), showing a higher incidence of pain in males in these moments. At 18 hours, one female has referred mild pain as compared to 10 males, with a high incidence of pain among males. At 24 hours, two females have referred moderate pain and no one has referred mild pain, while among males there has been one case of moderate and nine cases of mild pain, showing a higher incidence of mild or moderate pain among males. Two females have referred pain at first postoperative evacuation as compared to eight males. At 12 post-blockade hours, all patients had perineal anesthesia; at 18 hours, 22 patients, being 15 females and 7 males, indicating lower probability of blockade for males in this period. At 24 hours, blockade persisted in 12 patients, being 5 females and 7 males, with no statistically significant difference in this moment.

Analgesia duration was 15 to 20 hours in 10 patients; 21 to 25 hours, in 14 patients; 26 to 30 hours, in 10 patients; and more than 31 hours in 1 patient, with mean duration of 23.77 hours. Postoperative analgesia was optimal for 23 patients; satisfactory, for 5 patients, and unsatisfactory, for seven patients. There has been no need for postoperative analgesia in 23 patients. First evacuation was approximately 30 hours after bilateral pudendal nerves block; 10 patients have referred pain at evacuation, while 25 patients have not. Analgesic technique was considered excellent by 32 patients and only 3 male patients have considered it satisfactory due to penile anesthesia. There has been no difference in level of satisfaction between genders $(p=0.10)$ (Tables III and IV).
Table III - Analgesia Duration, Perineal Anesthesia Duration, Time for First Evacuation, Pain at First Evacuation and Satisfaction with the Technique

\begin{tabular}{lc}
\hline Analgesia duration $(\mathrm{h})^{*}$ & $23.77 \pm 4.49$ \\
Perineal analgesia duration $(\mathrm{h})^{*}$ & $20.21 \pm 2.79$ \\
Time $1^{\text {st }}$ evacuation $(\mathrm{h})^{*}$ & $29.60 \pm 4.84$ \\
First evacuation & 10 \\
$\quad$ Painful & 25 \\
$\quad$ Painless & \\
Satisfaction with the technique & 32 \\
$\quad$ Excellent & 3 \\
Satisfactory & 0 \\
\hline Poor &
\end{tabular}

*Values expressed in Mean $\pm S D$

Table IV - Analgesia Duration and Number of Patients

\begin{tabular}{cc}
\hline Analgesia Duration $(\mathrm{h})$ & $\mathrm{N}^{\circ}$ of Patients \\
\hline 16 & 1 \\
17 & 1 \\
18 & 1 \\
19 & 6 \\
20 & 1 \\
21 & 3 \\
22 & 1 \\
23 & 1 \\
24 & 3 \\
25 & 6 \\
26 & 3 \\
28 & 3 \\
29 & 1 \\
30 & 3 \\
36 & 1 \\
$23.77 \pm 4.49$ & \\
\hline
\end{tabular}

\section{DISCUSSION}

External hemorrhoidal thrombosis is probably one of the most common anorectal emergency diagnoses and surgery is the treatment, of choice. In our study, postoperative analgesia with bilateral pudendal nerves block under spinal anesthesia has resulted in mean 23.77 hours analgesia with low need for opioids and 23 patients without rescue medication.

Hemorrhoidectomy is a short procedure, however extremely painful, and few studies are directed toward postoperative analgesia ${ }^{17}$. Major pain factors are related to surgical proce- 
dure. Milligan-Morgan technique (open) seems to be much more painful as compared to Reis Neto semi-open hemorrhoidectomy or even to the closed procedure ${ }^{18}$. Some authors use posterior perineal block for anesthesia and postoperative analgesia.

There are several variants of initial descriptions ${ }^{11,19,20}$. Differences refer to technical details, equipment and anesthetic drugs. It is also necessary to differentiate pararectal infiltration from perineal block. Pararectal (or perineal) infiltration is a superficial posterior perineal block often used as single technique ${ }^{21}$. Our study has used bilateral pudendal nerves block oriented by nerve stimulator, resulting in perineal anesthesia for 20.21 hours and showing that blockade may be used as single technique for the proposed procedure. It was not our proposal to evaluate differences in the incidence of pain between genders. However, there has been higher incidence of pain among males as compared to females in all evaluated periods and during first evacuation. These data shall be the object of further studies.

All anesthetic drugs have already been used for posterior perineal block: bupivacaine, lidocaine, mepivacaine, ropivacaine, associated or not, with or without epinephrine, and in volumes varying from 20 to $50 \mathrm{~mL}$. Lidocaine or mepivacaine administered to 400 patients have induced analgesia for 5 hours in $31.5 \%$, for more than 10 hours in $48.5 \%$ and for more than 15 hours in $9.2 \% ; 3.2 \%$ had no need for analgesics ${ }^{11}$. One to three $\mathrm{mL}$ of $0.5 \%$ bupivacaine injected in the base of each hemorrhoid 10 minutes before incision have allowed a painless period 10 times longer as compared to control group ${ }^{10}$. The injection of $20 \mathrm{~mL}$ of $0.25 \%$ bupivacaine with levogyrous enantiomeric excess of $50 \%$ (S75:R25) in each pudendal nerve has promoted mean 23.77 hours analgesia. A different publication ${ }^{16}$ evaluating the same solution with twice the concentration $(0.5 \%)$ in the brachial plexus has not evaluated analgesia duration, thus not allowing the comparison of results.

Spinal anesthesia leads to clinical vesical function disorders due to the interruption of micturition reflex. Bladder function remains imperfect until the blockade has regressed to the $3^{\text {rd }}$ sacral segment in all patients ${ }^{22}$. With long lasting anesthetics, accumulated volume may exceed the cystometric capacity of the bladder ${ }^{17}$. For this reason, spinal anesthesia in our study was induced with $50 \mathrm{mg}$ of $2 \%$ isobaric lidocaine, in an attempt to prevent urinary retention caused by the anesthetic technique, which was confirmed by spontaneous micturition in all patients. This shows that bilateral pudendal nerves block has resulted in perineal anesthesia for approximately 20 hours without preventing spontaneous micturition.

This pilot study has used bilateral pudendal nerves block with the aid of peripheral nerve stimulator to control postoperative pain of patients submitted to hemorrhoidectomy under spinal anesthesia. The study has its limitations because analgesia results were not compared to a different method.

Based on our results, we consider that bilateral pudendal nerves block with peripheral nerve stimulator for postoperative analgesia with $0.25 \%$ S75:R25 bupivacaine may be indicated for all anorectal procedures, provided no previous infection is present. With this technique, analgesia is effective, without local or systemic complications, making first evacuation less painful and without side effects, such as urinary retention. In the future, controlled and comparative studies may show whether this technique should be the first option for hemorrhoidectomy analgesia. Mean perineal anesthesia duration of 21.20 hours shall induce further studies with pudendal nerves block and nerve stimulator.

\section{ACKNOWLEDGMENT}

We acknowledge professor Manoel Antônio Pereira Alvarez for suggesting the study.

\section{REFERÊNCIAS - REFERENCES}

01. Johanson JF, Sonnenberg A - The prevalence of hemorrhoids and chronic constipation. An epidemiologic study. Gastroenterology, 1990;98:380-386.

02. Smith LE - Ambulatory surgery for anorectal diseases: an update. South Med J, 1986;79:163-166.

03. MacRae HM, McLeod RS - Comparison of hemorrhoidal treatment modalities. A meta-analysis. Dis Colon Rectum, 1995;38: 687-694.

04. Kilbride M, Morse M, Senagore A - Transdermal fentanyl improves management of postoperative hemorrhoidectomy pain. Dis Colon Rectum, 1994;37:1070-1072.

05. Macario A, Weinger M, Carney S et al - Which clinical anesthesia outcome are important to avoid? The perspective of patients. Anesth Analg, 1999;89:652-658.

06. Rawal N, Hylander J, Nydahl PA et al - Survey of postoperative analgesia following ambulatory surgery. Acta Anaesthesiol Scand, 1997;41:1017-1022.

07. Goldstein ET, Williamson PR, Larach SW - Subcutaneous morphine pump for postoperative hemorrhoidectomy pain management. Dis Colon Rectum, 1993;36:439-446.

08. Chiu JH, Chen WS, Chen $\mathrm{CH}$ et al - Effect of transcutaneous electrical nerve stimulation for pain relief on patients undergoing hemorrhoidectomy: prospective, randomized, controlled trial. Dis Colon Rectum, 1999;42:180-185.

09. Coloma M, Duffy LL, White PF et al - Dexamethasone facilitates discharge after outpatient anorectal surgery. Anesth Analg, 2001;92:85-88.

10. Jirasiritham S, Tantivitayatan K, Jirasiritham S - Perianal blockage with $0.5 \%$ bupivacaine for postoperative pain relief in hemorrhoidectomy. J Med Assoc Thai, 2004;87:660-664.

11. Gabrielli F, Cioffi U, Chiarelli M et al - Hemorrhoidectomy with posterior perineal block: experience with 400 cases. Dis Colon Rectum, 2000;43:809-812.

12. Capelhuchink P, Ju LY, Carvalho F et al - Hemorroidectomia ambulatorial com bloqueio da fossa isquiorretal com lidocaína e/ou morfina. Rev Bras Coloproct, 2002;22:77-81.

13. Terminologia Anatômica. Terminologia Anatômica Internacional. Comissão Federativa da Terminologia Anatômica. $1^{\text {a }}$ Ed, São Paulo, Editora Manole Ltda, 2001;169. 
14. Simonetti MPB, Batista RA, Ferreira FMC - Estereoisomeria: a interface da tecnologia industrial de medicamentos e da racionalização terapêutica. Rev Bras Anestesiol, 1998;48: 390-399

15. Cox CR, Faccenda KA, Gilhooly $C$ et al - Extradural $\mathrm{S}(-)$ bupivacaine: comparison with racemic RS-bupivacaine. $\mathrm{Br}$ J Anaesth, 1998:80:289-293

16. Sato RT, Porsani DF, Amaral AGV et al - Bupivacaína racêmica a $0,5 \%$ e mistura com excesso enantiomérico de $50 \%$ (S75:R25) a $0,5 \%$ no bloqueio do plexo braquial para cirurgia ortopédica. Estudo comparativo. Rev Bras Anestesiol, 2005;55:165-174

17. Moiniche S, Kehlet $H$, Dahl JB - A qualitative and quantitative systematic review of preemptive analgesia for postoperative pain relief: the role of timing analgesia. Anesthesiology, 2002; $96: 725-741$.

18. Conférence de consensus: traitement de la maladie hémorroïdaire. Ann Chir, 2001;126:845-849.

19. Nivatvongs $S$ - An improved technique of local anesthesia for anorectal surgery. Dis Colon Rectum, 1982;25:259-260.

20. Nivatvongs $S$ - Technique of local anesthesia for anorectal surgery. Dis Colon Rectum, 1997;40:1128-1129.

21. Rowsell M, Bello M, Hemingway DM - Circumferential mucosectomy (stapled haemorrhoidectomy) versus conventionnal haemorrhoidectomy: randomised controlled trial. Lancet, 2000;355:779-781.

22. Kamphuis ET, lonescu TI, Kuipers PW et al - Recovery of storage and emptying functions of the urinary bladder after spinal anesthesia wit lidocaine and with bupivacaine in men. Anesthesiology, 1998;88:310-316.

\section{RESUMEN}

Imbelloni LE, Beato L, Beato C, Cordero JA, Souza DD - Analgesia Pos-Operatoria con Bloqueo Bilateral del Nervio Pudendo con Bupivacaína S75:R25 a 0,25\%. Estudio Piloto en Hemorroidectomia bajo Régimen Ambulatorial

JUSTIFICATIVA Y OBJETIVOS: La hemorroidectomia puede ser realizada bajo varias técnicas anestésicas y en régimen ambulatorial. El dolor pos-operatorio es intenso y puede atrasar el retorno para el hogar. El objetivo de este estudio fue evaluar las ventajas y la realización del bloqueo bilateral de los nervios pudendos para analgesia pos-operatoria en hemorroidectomias.

MÉTODO: El bloqueo bilateral de los nervios pudendos con bupivacaína S75:R25 a 0,25\% fue realizado con estimulador de nervios en 35 pacientes sometidos a la hemorroidectomia bajo raquianestesia. Fueron evaluadas intensidad del dolor, duración de la analgesia, analgesia de demanda y eventuales complicaciones relacionadas a la técnica. Los datos fueron evaluados a las 6, 12, 18, 24 y 30 horas después del término de la intervención quirúrgica.

RESULTADOS: En todos los pacientes, fue logrado éxito con la estimulación de ambos los nervios pudendos. En ningún momento de la evaluación ocurrió dolor intenso. Hasta 12 horas después del bloqueo, todos los pacientes presentaron anestesia en la región perineal; con 18 horas, 17 pacientes y 24 horas, 10 pacientes $A$ analgesia pos-operatoria fue óptima en 18 pacientes; satisfactoria, en cinco pacientes; e insatisfactoria, en siete pacientes. La duración media de la analgesia fue de 23,77 horas. No ocurrieron alteraciones de la presión arterial, de la frecuencia cardiaca, ni fueron observados náuseas o vómitos. Todos los pacientes tuvieron micción espontánea. Ninguna complicación local o sistémica fue relacionada al anestésico local. Veintisiete pacientes clasificaron de excelente la técnica de analgesia y apenas tres pacientes del sexo masculino quedaron satisfechos, justificado por la anestesia en el pene.

CONCLUSIONES: El bloqueo bilateral de los nervios pudendos, orientado por estimulador de nervios proporciona una analgesia de excelente calidad, con baja necesidad de opioides, sin complicaciones local o sistémica y sin retención urinaria. Estudios controlados permitirán demostrar si esta técnica debe ser la primera opción para la analgesia en hemorroidectomias. La permanencia de anestesia perineal por 20,21 horas deberá inducir nuevos trabajos con el bloqueo de los nervios pudendos orientado por estimulador para el acto quirúrgico. 